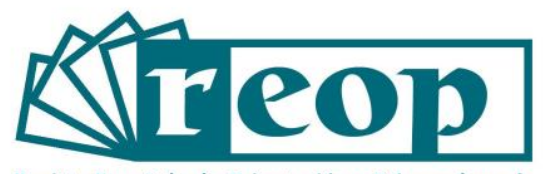

Revista Española de Orientación y Psicopedagogía

\title{
DESARROLLO DE LA ACTITUD EMPRENDEDORA: ESTUDIO CUALITATIVO DE UN MODELO DE FORMACIÓN UNIVERSITARIA
}

\author{
DEVELOPING AN ENTREPRENEURIAL ATTITUDE: QUALITATIVE STUDY OF A \\ UNIVERSITY TRAINING MODEL
}

\author{
Orlando Llanos-Contreras \\ Universidad Católica de la Santísima Concepción. Facultad de Ciencias Económicas y \\ Administrativas. Departamento de Administración. Concepción, Chile \\ Melany Hebles ${ }^{1}$ \\ Universidad Católica de la Santísima Concepción. Facultad de Ciencias Económicas y \\ Administrativas. Departamento de Administración. Concepción, Chile \\ Concepción Yániz-Álvarez-De-Eulate \\ Universidad de Deusto. Facultad de Psicología y Educación. Departamento de Didáctica y \\ Desarrollo Curricular. Bilbao, España
}

\section{RESUMEN}

Desarrollar la actitud emprendedora, componente esencial de la competencia para innovar y emprender, es un objetivo importante de la orientación para el desarrollo profesional propio de la formación universitaria, y una contribución para impulsar el desarrollo económico y social. En esta investigación se estudian las variables principales para la formación de dicha actitud, utilizando un estudio exploratorio basado en un caso único de implementación de un programa universitario de formación de competencias en innovación y emprendimiento llevado a cabo con estudiantes de ingeniería, salud, ciencias sociales, ciencias y economía. Se analiza información proveniente de profesorado, coordinación del programa y estudiantes obtenida con entrevistas, cuestionarios y grupos focales, siguiendo un proceso inductivo-deductivo con ayuda del software MAXQDA12. Este trabajo contribuye al conocimiento del tema: (1) proponiendo la creación de un ecosistema de aprendizaje como el elemento más relevante en la formación de la actitud emprendedora, (2) identificando los elementos que componen dicho ecosistema (programa formativo, estudiantes y

1 Correspondencia: Melany Hebles. Correo-e: mhebles@ucsc.cl 
ecosistema de emprendimiento regional) y cómo interactúan, y (3) destacando el carácter sistémico del proceso que forma la actitud emprendedora. Como conclusión se observa que la implementación de este programa impulsa la orientación para el desarrollo profesional dentro de la universidad, y de esta con el entorno público y privado que dan forma a un ecosistema de emprendimiento, lo que contribuye con la comprensión del papel que pueden jugar las universidades en la orientación para el desarrollo profesional mediante procesos formativos en emprendimiento y el fortalecimiento de ecosistemas de emprendimiento regional.

Palabras clave: Orientación profesional, actitud emprendedora, ecosistema de aprendizaje, ecosistema de emprendimiento, modelo de formación, innovación.

\section{ABSTRACT}

Developing an entrepreneurial attitude, which is an essential component for innovation and entrepreneurship, is an important objective for career guidance in Higher Education, and a contribution to promote economic and social development. This research examines the main variables to form this attitude, using an exploratory study based on a single case of implementation of a university program to develop innovation and entrepreneurship skills, carried out with students of engineering, health, social sciences, sciences, and economics. Information coming from teachers, program coordination and students is analyzed. The information, obtained through interviews, questionnaires, and focus groups, is analyzed following an inductive-deductive process with the aid of the MAXQDA12 software. This work contributes to the knowledge in the area: (1) by proposing the creation of a learning ecosystem as the most relevant element in the development of an entrepreneurial attitude, (2) by identifying the components of this ecosystem (formative program, students, and ecosystem of regional entrepreneurship) and how they interact, and (3) emphasizing the systemic character of the process that forms the entrepreneurial attitude. As a conclusion, the implementation of this program promotes guidance for professional development within the university, with the public and private environment that shape an entrepreneurial ecosystem, contributing to the understanding of the role that universities can play in career guidance through entrepreneurial training processes and the strengthening of regional entrepreneurial ecosystems.

Key Words: career guidance, entrepreneurial attitude, learning ecosystem, entrepreneurial ecosystem, training model, innovation.

\section{Cómo citar este artículo:}

Llanos-Contreras, O., Hebles, M. y Yániz-Álvarez-De-Eulate, C. (2021). Desarrollo de la actitud emprendedora: estudio cualitativo de un modelo de formación universitaria. Revista Española de Orientación y Psicopedagogía, 32(3), 112-131. https://doi.org/10.5944/reop.vol.32.num.3.2021.32560 


\section{Introducción}

Desde hace dos décadas se observa un interés creciente por incorporar la formación de la actitud emprendedora en los procesos formativos (Huq y Gilbert, 2017), componente esencial de la competencia para innovar y predictora de la actividad emprendedora (Harris y Gibson, 2008) relevante para impulsar el desarrollo económico y social (Alonso-Dos-Santos y Llanos-Contreras, 2018; Duarte y Tibana, 2009; Raposo y do Paco, 2011). El importante papel de la educación superior en esta formación (Hägg y Kurczewska, 2020; Keinanen y Kairisto-Mertanen, 2019) ha influido en la implementación de programas para lograrla (Kuratko, 2005; Vanevenhoven, 2013). Frecuentemente están orientados a mejorar características parciales como la autoconfianza, la creatividad y la capacidad de trabajar en equipo (Sánchez-García y Hernández-Sánchez, 2016), prescindiendo de aspectos organizacionales y del entorno que, junto con el perfil de ingreso del alumnado (Bae et al., 2014; Hebles, Llanos-Contreras y Yaniz-Alvarez-de-Eulate, 2019) y las características de los modelos de intervención psicopedagógica que se implementan, son importantes en el desarrollo de la actitud para emprender. Hägg y Kurczewska (2020) aluden a la insuficiente presencia de la orientación en estos procesos, de manera que el alumnado esté debidamente apoyado a lo largo de los mismos. Esto podría explicar los resultados contradictorios del impacto de estos programas en el desarrollo de la actitud emprendedora encontrados (Nabi et al., 2017). Asimismo, se sabe poco sobre cómo interactúan los elementos asociados a cada uno de estos factores. Palomares-Montero et al. (2019) llaman la atención sobre la escasez de estudios que tengan en cuenta la orientación profesional en la formación emprendedora y sobre la aún mayor escasez de propuestas que incorporen una orientación integral en esta formación.

En esta investigación se plantea la siguiente pregunta: ¿Cómo influyen el perfil de ingreso de los estudiantes, el ecosistema de emprendimiento y el modelo de intervención en la formación de la actitud emprendedora de estudiantes de pregrado?

Para responder a esta pregunta, se desarrolla un estudio exploratorio sobre la implementación de un programa de formación de competencias en emprendimiento e innovación en una universidad.

\section{La formación de la actitud emprendedora}

Emprender es desarrollar acciones de innovación y creación para responder a demandas y desafíos del entorno (Alda, 2010). La actitud emprendedora se refiere al componente emocional de emprender (Hebles et al., 2019) e incluye la disposición de las personas para actuar proactivamente, innovar, asumir riesgos, autocontrolarse, desarrollar autoconocimiento y seguridad en sí mismo, definir acciones para el logro de metas, detectar oportunidades en el entorno y proponer soluciones a desafíos, entre otros (Díaz-García et al., 2015; Krauss, 2011; Raposo et al., 2008).

Las universidades han liderado la implementación de programas para promover que las y los graduados vean en el emprendimiento una oportunidad atractiva para su desarrollo profesional y personal (Morris et al., 2013; Sánchez, 2011). Se asume que los aspectos curriculares y metodológicos, y la coherencia del diseño con los objetivos juegan un papel central en esta formación (Nabi et al., 2017).

Las propuestas son heterogéneas (Kassean et al., 2015; Morris et al., 2013). Algunas utilizan la elaboración de planes de negocio, incluyendo la identificación de oportunidades, el desarrollo de ideas (prototipos), la estimación y búsqueda de los recursos requeridos para desarrollar el proyecto, y la implementación del mismo (Neck y Greene, 2011). Otras se centran en la 
experimentación y la flexibilidad desarrollando la creatividad y la disposición a construir relaciones y trabajar en equipos (Azqueta y Naval, 2019; Chandler et al., 2011; García-Rodríguez et al., 2016). En todas ellas se reconoce la importancia de los equipos de profesores para la aplicación exitosa de los métodos (Raposo y do Paco, 2011); y se echa en falta conocimiento sobre cuáles son las técnicas más efectivas y por lo tanto es importante generar más conocimiento riguroso sobre ello (Holmgren et al., 2004).

Por último, la mayoría de las propuestas de intervención tienen una importante orientación al aprendizaje experiencial, que aporta valor psicopedagógico a las mismas. Sin embargo, este potencial puede desaprovecharse en la medida en que no se acompaña de suficiente orientación. Los estudiantes universitarios necesitan experimentar también un fuerte componente de orientación en esta formación (Hägg y Kurczewska, 2020), necesidad que confronta a los docentes con su función orientadora.

\section{Perfil del alumnado y actitud emprendedora}

Asumiendo la idoneidad de las universidades para transmitir la cultura de emprendimiento (Duarte y Tibana, 2009), surge el interés por los mecanismos, recursos y restricciones que determinan el éxito en el desarrollo de la actitud emprendedora.

El perfil de competencias del alumnado que accede a este tipo de programas podría afectar de manera determinante al éxito en el desarrollo de la actitud emprendedora (Fayolle y Gailly, 2015) y conocerlo permite plantear la formación de manera más efectiva (Keinanen y KairistoMertanen, 2019). Cada estudiante presenta diferencias en habilidades de liderazgo, gestión de conflictos, comunicación, resolución de problemas y toma de decisiones, así como en conocimiento, disciplinas de procedencia y experiencias previas (Lans et al., 2013; Popov et al., 2012) o perfil de motivación para el emprendimiento (Suárez-Ortega et al., 2020).

Se han identificado factores de heterogeneidad importantes, pero falta conocer cómo influyen en la formación de la actitud emprendedora y cómo gestionar las diferencias en la implementación de estos programas, incorporando un sistema de orientación personal y profesional integrado.

\section{Ecosistemas de emprendimiento y formación de actitudes emprendedoras}

Los aspectos organizativos y contextuales afectan a la formación de actitudes (Ajzen y Fishbein, 2000). La interacción con agentes externos, tanto del sector privado como público (Schmidt y Molkentin, 2015) es una fuente de aprendizaje; y las personas que participan en un proceso emprendedor necesitan habilidades para explorar el entorno y generar ideas que luego puedan ser implementadas (Alda-Varas et al., 2012).

Este conjunto de actores en interacción constituye un ecosistema de emprendimiento, en el cual las universidades juegan un papel formativo y comunicativo importante (Radharamanan y Juang, 2012; Stam, 2015). El desarrollo de ecosistemas de formación del talento emprendedor se ve favorecido por la interacción de la institución educativa con su entorno social e industrial (Cao y Zhou, 2018); el sector privado apoya el desarrollo de modelos de empresa y negocios; y el sector público facilita el cumplimiento de aspectos normativos y el acceso a financiación pública (Backs et al., 2019; Guerrero et al., 2016). 
Las universidades proporcionan un espacio para la cooperación entre estudiantes y empresariado, y son consideradas por las instituciones públicas plataformas de confianza en las que pueden cooperar y coordinarse (Guerrero y Urbano, 2012).

Todas las universidades manifiestan interés por esta formación, pero solo algunas desarrollan ecosistemas de emprendimiento como característica distintiva. Este grupo logra vínculos más sólidos universidad-empresa-estado, generando mayor impacto económico y social (Guerrero et al., 2014).

Dicho ecosistema puede proporcionar soporte al desarrollo de la actitud emprendedora del alumnado. Sin embargo, este es un tema inexplorado y por lo tanto no se conoce cómo se forman dichos ecosistemas de aprendizaje y tampoco cuáles son los mecanismos a través de los cuales se forma la actitud emprendedora.

\section{Método}

Con el objetivo de conocer cómo influyen el ecosistema de emprendimiento, el perfil de ingreso del alumnado y el diseño del programa en la formación de la actitud emprendedora se ha estudiado el desarrollo de la actitud emprendedora en estudiantes universitarios y la contribución a la adquisición de la competencia para emprender, de la implementación de un programa formativo de competencias en innovación y emprendimiento utilizando técnicas de investigación cualitativa. Se trata de un estudio de caso único (Yin, 2014), seleccionado porque posibilitaba contar con información sobre las interacciones de directivas/os, profesorado y estudiantes sobre la implementación del modelo: métodos de enseñanza, características del funcionamiento de los equipos de trabajo, las técnicas de evaluación, las estructuras de soporte y el seguimiento del trabajo de las y los estudiantes involucrados (Woodside, 2010). Los estudios de caso se han utilizado frecuentemente en ciencias sociales, siendo valiosos en los campos orientados a la práctica como la educación (Starman, 2013). Para esta investigación, se estudió el caso en un marco de tiempo ajustado y fue diseñado para el desarrollo de un proceso de teorización y no con el objetivo de evaluar la implementación del programa en cuestión.

\section{Descripción del programa}

El programa está dirigido a estudiantes de las facultades de ingeniería, salud, ciencias sociales, ciencias y economía, y tiene como objetivo fortalecer sus intereses por esta área y desarrollar diversos aspectos de la competencia para innovar y emprender. La universidad busca crear un ecosistema de aprendizaje que proporcione a los estudiantes soporte académico, administrativo y de vinculación social, institucional y empresarial para que puedan generar proyectos de emprendimiento e innovación. Incluye seis cursos de carácter optativo, tres de emprendimiento (Emprender, Proyecto de Emprendimiento y Desafío Startup) orientados a apoyar el desarrollo de iniciativas de los estudiantes y de la comunidad, y otros tres de innovación (Innovación Social, Innovación en Productos/Servicios y Consultoría en Innovación) orientados a que promuevan iniciativas que den respuestas innovadoras con valor social. 


\section{Muestreo teórico y recolección de datos}

Este caso da acceso a información de alto valor sobre las características iniciales de un variado grupo de estudiantes interesados en el emprendimiento e innovación, y al diseño y la implementación de un proyecto formativo innovador.

Se obtuvo información de tres grupos de informantes clave: estudiantes, profesores y directivos involucrados en el diseño e implementación del proyecto (Yin, 2014), que aportan retroalimentación desde todos los puntos de vista participantes. Se recurrió principalmente a fuentes primarias, a través de entrevistas personales con profesores y encargados del proyecto, aplicación de cuestionarios y participación en grupos focales con estudiantes, y observación de reuniones de trabajo de profesores y directivos.

Para la selección de los informantes clave entre los profesores y encargados del proyecto se construyó una muestra teórica bajo el criterio cadena de referencia; se identificó al líder del proyecto y este referenció potenciales entrevistados, quienes a su vez recomendaron otros nombres que contaban con información que aportaría a los objetivos de la investigación (Hunter y Cooksey, 2004).

En el caso de los estudiantes, se solicitó a la totalidad de los inscritos en los cursos asociados al programa del segundo semestre de 2016 que respondieran al cuestionario, obteniéndose una respuesta del $23 \%$.

Para la realización de entrevistas grupales en grupos focales, la muestra se seleccionó por conveniencia incorporando alumnos disponibles para participar provenientes de cuatro de las carreras de la universidad (Woodside, 2010).

La Tabla 1 resume las principales actividades de recolección de datos primarios. 
Desarrollo de la actitud emprendedora...

Orlando Llanos-Contreras et al.

\section{Tabla 1}

Resumen de actividades de recolección de datos primarios

\begin{tabular}{|c|c|c|c|c|}
\hline Tipo & Docente/Directivo/Curso & Académico & Participantes & \\
\hline \multirow{6}{*}{$\begin{array}{l}\text { Entrevista } \\
\text { Individual }\end{array}$} & & $\overline{\mathrm{CC}}$ & 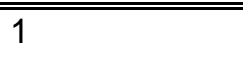 & \\
\hline & & SS & 1 & \\
\hline & Locente programa $5+1$ & FP & 1 & \\
\hline & & JE & 1 & \\
\hline & & JE & 1 & \\
\hline & Decano/director programa $\mathrm{E}+\mathrm{I}$ & $A U$ & 1 & \\
\hline \multirow[t]{2}{*}{ Tipo } & Docente/Directivo/Curso & Curso asociado & Participantes & $\begin{array}{l}\text { Total, alumnos } \\
\text { por curso }\end{array}$ \\
\hline & & "Innovación Social & (26 & 53 \\
\hline \multirow[t]{2}{*}{$\begin{array}{l}\text { Entrevista } \\
\text { Grupales }\end{array}$} & Estudiantes & Desafío Start Up & 6 & 31 \\
\hline & & Club emprende & 6 & 10 \\
\hline \multirow[t]{2}{*}{ Tipo } & Docente/Directivo/Curso & $\begin{array}{l}\text { Numero } \quad \text { de } \\
\text { encuestas } \\
\text { enviadas }\end{array}$ & $\begin{array}{l}\text { Encuestas } \\
\text { respondidas }\end{array}$ & \\
\hline & $\begin{array}{l}\text { Innovación en productos y } \\
\text { servicios }\end{array}$ & 18 & 5 & \\
\hline \multirow[t]{3}{*}{ Encuestas } & Proyecto de emprendimiento & 16 & 5 & \\
\hline & Consultoría en innovación & 25 & 4 & \\
\hline & Desafío Start Up & 30 & 6 & \\
\hline \multicolumn{3}{|c|}{ Porcentaje encuestas respondidas } & $22,47 \%$ & \\
\hline
\end{tabular}

Fuente: Elaboración propia

\section{Análisis de datos}

Para el análisis de datos se siguió un proceso inductivo/deductivo (Thomas, 2006). De manera inductiva se realizó una categorización abierta para agrupar fragmentos relevantes del discurso en categorías. Mediante análisis deductivo se agruparon las categorías en temas, según la literatura base del estudio para identificar patrones y desarrollar un modelo de teorización. Como normalmente ocurre en este tipo de estudios, el primer análisis de la información se hizo casi en paralelo con la recolección de los datos. Esto introduce un nivel aceptado de sesgo al estudio, que se controló por medio del uso de notas de campo y de protocolos estandarizados para entrevistas (Báez y De Tudela, 2007). Para incrementar la validez y confiabilidad, se crearon bases de datos con toda la información recolectada antes y durante el proceso de análisis. Esto además con el propósito de mejorar la trazabilidad de la información. 


\section{Figura 1}

\section{Sistema de códigos}

\begin{tabular}{|lll}
\hline Códigos primarios & Códigos secundarios & Dimensiones Agregadas \\
\hline
\end{tabular}

Aumento de la iniciativa
Participación en actividades de emprendimiento
Movilización de equipos para el logro de objetivos

Variabilidad en la vitalidad del logro de objetivos
Vacion

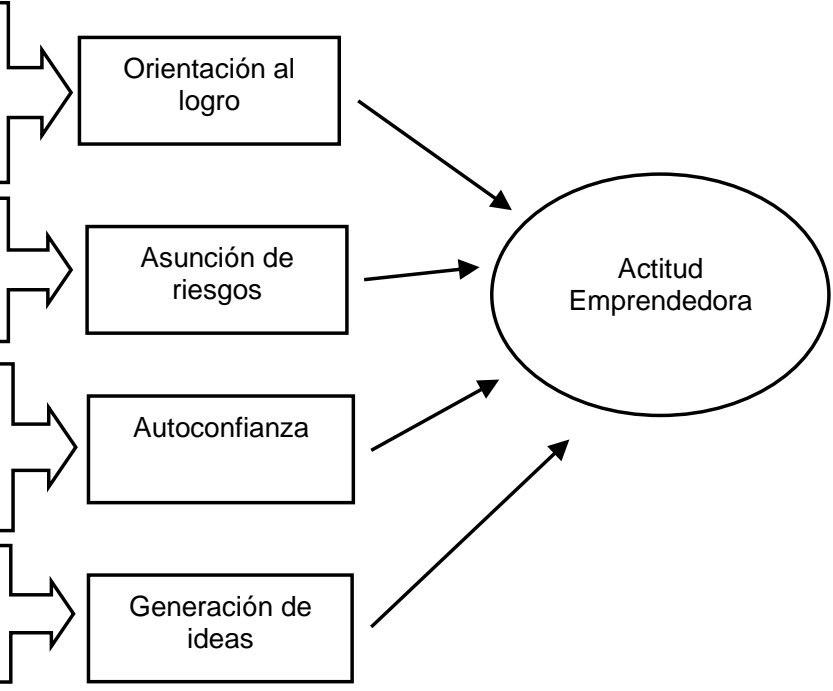

Más entusiasmo en la generación de ideas

Aumento en la identificación de problemas del entorno

Búsqueda constante de soluciones

Disposición a experimentar

Menor temor al fracaso

Estar abierto al error y a equivocarse
Mayor autoconocimiento

Estudiantes más reflexivos

Mayor seguridad en la generación de propuestas

Desarrollo del autoaprendizaje

Heterogeneidad en motivaciones de ingreso al programa Heterogeneidad de carreras de procedencia

Heterogeneidad del entorno social del estudiante

Heterogeneidad de las habilidades sociales y emocionales

Brecha en actitud emprendedora de los estudiantes Brecha de personalidad de los estudiantes

Capacidad de mantener la motivación en los cursos del programa

Soporte administrativo para implementación del modelo

Contar con vínculo institucional con la comunidad

Capacidad de articulación institucional
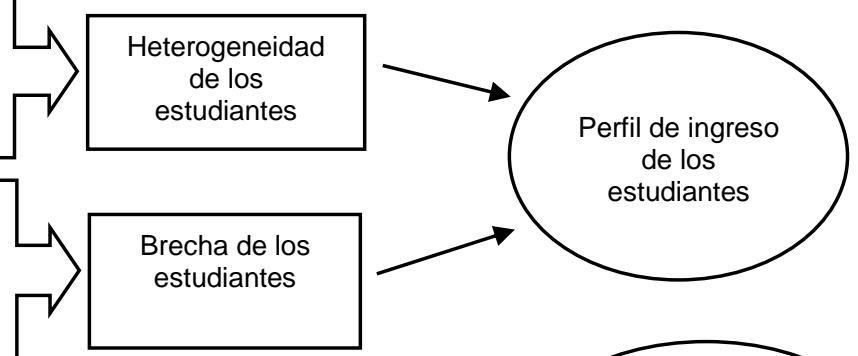

Ecosistema

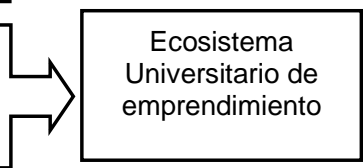

Institucionalidad que fomenta el emprendimiento

Disposición público-privada-universidad para vincularse

Fomento al emprendimiento en agenda pública
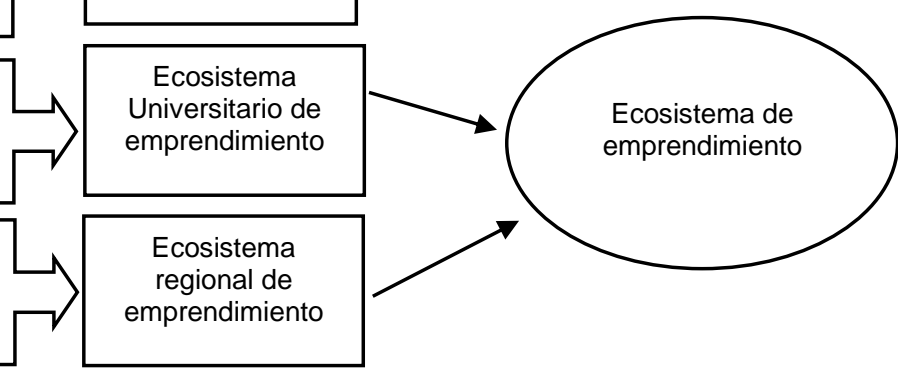

Articulación de cursos del programa de intervención

Número excesivo de estudiantes por curso

Articulación para promover aprendizajes diversos

Mejoramiento continuo de métodos
Énfasis en la metodología colaborativa
Evaluación orientada al aprendizaje

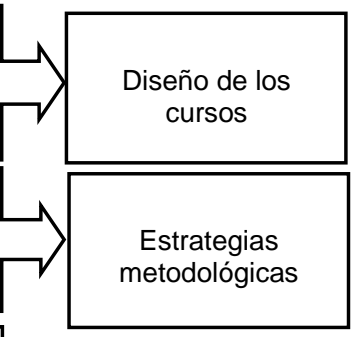

Buena calidad de la relación profesor alumno

Trabajo colaborativo entre profesores del programa

Consolidación del equipo

Potencia del equipo

Formación en enseñanza de emprendimiento del profesor
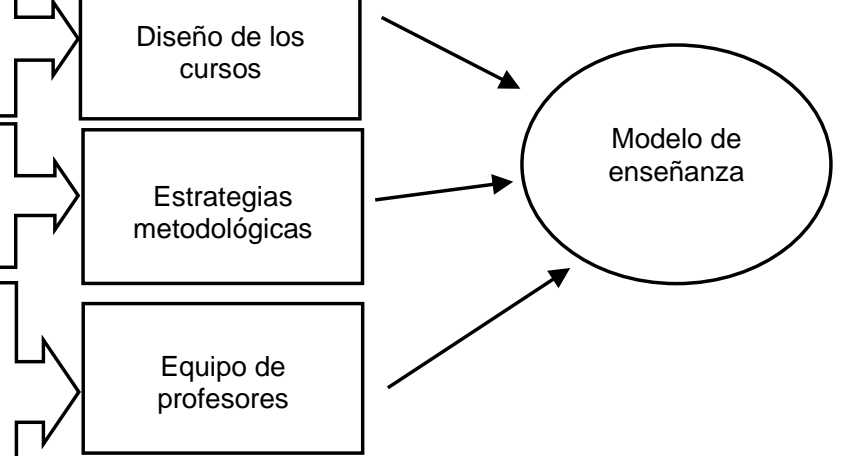

Fuente: Elaboración propia 
Para disminuir el sesgo propio del análisis cualitativo se usaron distintas técnicas de triangulación de información y aparejamiento de patrones discursivos proveniente de los distintos entrevistados y fuentes de información (De Massis y Kotlar, 2014); triangulación metodológica, usando dos técnicas de recolección diferentes, grupos focales y entrevistas individuales, para evaluar el mismo fenómeno; y triangulación del investigador, revisando el proceso de generación de códigos con dos investigadores externos, expertos en emprendimiento. El análisis se realizó en dos etapas: 1) análisis de cada uno de los grupos de actores relevantes del proyecto (estudiantes, profesores y directivos) y 2) análisis cruzado de los tres grupos involucrados en el proyecto. La información se procesó usando el programa MAXQDA12. La Figura 1 resume el sistema de códigos obtenido del análisis.

\section{Resultados}

El análisis multinivel permitió agrupar la información en un sistema de códigos (Figura 1): dimensiones de la actitud emprendedora desarrolladas con el programa de intervención; factores del ecosistema emprendedor; perfil de ingreso de los estudiantes; y modelo de intervención que actuaron como facilitadores y obstaculizadores de la implementación del programa. A partir del análisis de los patrones observados en la narrativa de directivos, profesorado y estudiantes, se identifican coincidencias y contrastes en el discurso. A continuación, se muestran los principales hallazgos.

\section{Dimensiones de la actitud emprendedora}

Profesorado y estudiantes consideran que con la implementación del modelo se desarrolló la orientación al logro, la asunción de riesgos, la autoconfianza y la generación de ideas, dimensiones todas ellas de la actitud emprendedora. Respecto a la orientación al logro se observó que el alumnado comenzó a involucrarse activamente en sus proyectos, buscando el desarrollo de redes de trabajo dentro y fuera de la universidad. Se mostró más proactivo, con mayor ambición y con ganas de hacer cosas, buscando activamente las oportunidades, sin esperar a que llegasen por sí solas.

“... ahora con esto (el curso) como que quiero hacerlo todo rápido, o tratar de alguna forma $u$ otra arreglar las cosas para que funcionen". (E. 141, II).

En los cursos se enfrenta a desafíos que implican estar constantemente buscando soluciones y propuestas. Con la orientación al logro de objetivos se desarrolla aún más la motivación por el emprendimiento. Esto mismo conlleva que el alumnado incremente su disposición a asumir riesgos incluyendo el hacer sacrificios que involucraban costos, disponerse a experimentar fracasos y equivocarse. El profesorado observó en las y los estudiantes un incremento en la tolerancia al riesgo, mayor disposición a experimentar y a atreverse a hacer cosas que antes no habrían hecho y menor vergüenza-temor al fracaso. 
"Pero me da la impresión de que están más sueltos y quizás por eso, porque van viendo que el contexto legitima justamente eso, la experimentación y el fracaso, como un insumo de información para los siguientes intentos, entonces como que se arriesgan un poquito más". ( $P$. II,48).

La narrativa también mostró una mayor autoconfianza. El profesorado señala que la implicación del alumnado en sus proyectos crece y se muestra más seguro en sus propuestas. Se observa un proceso de aprendizaje autónomo que le permite ir revisando constantemente su desempeño. En gran parte de las y los estudiantes se observa un trabajo de reflexión, reconocen sus propias habilidades y emociones, haciéndose más conscientes del proceso que están llevando a cabo.

"Creo que el curso me entregó muchas herramientas y la confianza de que se puede cumplir el sueño de algún negocio que se tenga en mente". (E. 102, IV).

El alumnado se reconoce con más capacidad de desarrollar ideas que antes. Reconoce que los aportes de estos cursos van moldeando sus ideas hasta diseñar un plan de trabajo para llevarlas a cabo. El profesorado confirma el incremento en la capacidad de generar ideas más creativas como consecuencia del trabajo colaborativo entre el alumnado.

“...y aparecían ideas que a mí me asombraban mucho, entonces para ellos también era positivo cuando miraban las caras de nosotros de asombro, como si, el curso está diseñado de tal forma que les empieza a intencionar en el hecho de darle valor a sus ideas, y no hace falta que sean garantías de logro". (P. II, 78).

\section{Características del modelo de enseñanza}

Las características del modelo de enseñanza destacan como elementos relevantes para explicar el éxito en la formación de la actitud emprendedora del alumnado. Profesorado y estudiantes coinciden en que las estrategias metodológicas, la estructura de los cursos y los equipos de profesorado son determinantes en el éxito del proceso formativo. Las personas entrevistadas señalan que las estrategias metodológicas son críticas para mejorar la capacidad de motivar al alumnado, construir confianza y capital social entre los distintos actores que participan en el proceso. El método colaborativo se considera eje transversal del programa formativo; y el trabajo en equipo, siguiendo la metodología colaborativa propuesta, esencial para el desarrollo exitoso de los prototipos y de los avances en los proyectos. El aspecto que más destaca es la cooperación, que se traduce en consideración de los aportes de otros y apoyo mutuo.

"Porque ellos tienen que apoyarse unos con otros, entonces yo apoyo tu proyecto y tú también tienes que apoyar el mío y se apoyan de distintas formas. Y la idea es que todos vayan apoyándose para que generen las redes". (P. IV,122).

El hecho de que el proyecto de intervención incorporara los cursos como "asignaturas complementarías" generó fluctuaciones en el compromiso de los estudiantes. Quienes realizaron los cursos iniciales y desarrollaron mayores niveles de compromiso continuaron seleccionando asignaturas más avanzadas de este programa en los siguientes semestres. Teniendo en cuenta este dato y el hecho de que podían elegir cualquier asignatura del programa sin ningún requisito previo, se sugiere que sería conveniente una mayor articulación entre las distintas asignaturas. De este modo se podría hacer un seguimiento mejor del alumnado con potencial para desarrollar su actitud emprendedora, y fortalecer la eficacia del programa.

“... ellos por un lado al vivir esta experiencia logran tenerla, la logran... yo estoy segura... no tendríamos que verlos después, yo no sé qué pasa con los alumnos que tomaron innovación y 
pasaron al otro curso, yo no sé qué pasa con esos alumnos porque los ve otros profesores, yo lo que veo es la experiencia que viven en el primero curso". (P. IV, 3).

El tercer factor relevante de las características del modelo de enseñanza fue el equipo de profesorado. Se destaca la importancia del trabajo colaborativo, la potencia del equipo y la formación en orientación para el emprendimiento. El trabajo colaborativo del profesorado se favorece con la multidisciplinariedad del equipo. El trabajo coordinado de varios profesores en la clase es uno de los temas más mencionados por los entrevistados, y repercute positivamente en la motivación de los estudiantes para las actividades de los cursos.

“...decirnos cómo podríamos emprender, cómo podíamos mejorar, él lo hace constantemente, entonces eso yo creo que es importante también para este tipo de cursos, porque ellos son como los guías, entonces yo por lo menos es una buena experiencia, la metodología me gusto...". (E. 14, III).

El equipo docente presenta un buen nivel de autoeficacia, señalando que todos aportan, que ninguno destaca sobre el resto y que, pese a que los tiempos para reunirse a planificar el trabajo son pocos, se consideran un equipo potente. Complementariamente se observa interés en los profesores por mejorar su capacitación en emprendimiento, ya que algunas de las metodologías que se implementan son nuevas para ellos.

"Bueno ha sido una muy buena experiencia, en términos de en lo personal en lo académico primero... en que se abre un espacio interno dentro de la universidad donde pueden convivir de verdad interdisciplinariamente distintos colegas que además parten desde una generosidad que encuentro yo que tiene este proyecto". (P. III, 2).

Factores del perfil de ingreso y ecosistema de emprendimiento que facilitan/obstaculizan la implementación del modelo de formación emprendedora

Las profesoras y profesores entrevistados destacan la heterogeneidad y la actitud emprendedora inicial como factores que pueden facilitar u obstaculizar el avance del alumnado en los cursos del programa de formación. Cada estudiante se diferencia por el entorno social de procedencia, la carrera que estudian, y sus habilidades sociales y emocionales. Profesorado y estudiantes valoran esta diversidad como un obstaculizador de la implementación del modelo que provoca resistencia para trabajar de manera colaborativa con personas desconocidas, de otras facultades o carreras. Paradójicamente, también consideran la heterogeneidad un facilitador de la formación al enriquecer el desarrollo de ideas, el trabajo colaborativo, la generación de redes y la complementariedad de las habilidades para la solución de problemas.

Porque al comenzar evidentemente vienen como perdidos porque hay compañeros de otras carreras, entonces vienen cohibidos, porque ellos no están acostumbrados a ir a INS con chicos de otras carreras". (P, III,6).

"Enriqueció el dialogo [la heterogeneidad de los estudiantes] y la conversación que se da entre ellos cuando están diseñando sus proyectos, también se ofrecen contrapuntos que no se tendrían creo yo desde individuos de una misma disciplina”. (P.II, 10).

La actitud emprendedora inicial se identifica a través de la intención emprendedora. Las diferencias en dicha intención influían directamente en los niveles de motivación y compromiso en los cursos. Quienes presentaban menos intención emprendedora mostraron menos motivación en los cursos iniciales del programa, mientras que en los cursos más avanzados sus niveles de motivación y compromiso crecieron. 
"Ya teníamos ese "espíritu emprendedor", porque este curso... yo me acuerdo de que le pregunté al profesor cuál era la intención de este curso y me decía que era para tomar riesgos, o sea tener esa actitud y nosotros ya teníamos ese "espíritu emprendedor". (E. 13, III).

En relación con el ecosistema de emprendimiento se identifican dos grupos de códigos: (1) importancia del ecosistema regional de emprendimiento y (2) ecosistema universitario de emprendimiento.

Directivas/os, profesorado y estudiantes señalan que el hecho de que el ecosistema regional de emprendimiento se haya convertido en un tema relevante para el país y la región, ha facilitado el desarrollo de las actividades académicas vinculadas al proyecto. Las/os estudiantes reconocen, además, que la proliferación de fondos concursables institucionales disponibles es un aliciente que les motiva para considerar la opción de emprender como una oportunidad de desarrollo profesional futura.

"Ha sido facilitador también internamente el hecho de que el emprendimiento en el país, en la región sea como un tema de moda y que por lo tanto no sea tampoco tan ajeno a los estudiantes, lo están observando en afiches, en la radio, están expuestos a temáticas de emprendimiento constantemente y por lo tanto de alguna manera esto les entusiasma a los estudiantes". (D. II.4).

"Que hoy en día el mercado laboral está súper complicado, y el emprendimiento es una buena instancia para poder generar empleo y también obtener recursos, que al final es lo que nos mueve". (E. 87, III).

La buena disposición de instituciones públicas y privadas para vincularse con la Universidad es también un factor facilitador. Estos vínculos facilitan la articulación teórico-práctica, y permiten que el alumnado desarrolle sus habilidades y motivación emprendedora a través del desarrollo de proyectos de interés para la comunidad.

"Tenemos el apoyo de las instituciones, municipalidades, instituciones sociales, que les interesa que la universidad los apoye y que los estudiantes los apoyen entonces en ese sentido cuando los cursos involucran que los estudiantes tienen que relacionarse con municipalidades, empresas, emprendedores externos existe ese apoyo". (D. II.4).

Se reconoce que el ecosistema de emprendimiento universitario se ha formado lentamente con la implementación del proyecto de intervención. Además de la implementación de los seis cursos, se han organizado concursos de emprendimiento para estudiantes, trabajos de vinculación con la comunidad y se ha creado un club de innovación. Profesorado y directivos destacan la importancia del proyecto para el desarrollo de una cultura que promueve un ambiente de aprendizaje y da soporte al desarrollo de nuevas ideas y proyectos. El alumnado valora positivamente la incorporación de estos elementos para el proceso formativo.

"La conclusión que uno saca es que mientras más se desarrolle el ecosistema, y vengan emprendedores, empresarios, gente de municipalidades, nuestros estudiantes estén participando en ferias, distintas actividades, se genera más motivación”. (D. II.10).

"Creo que es bueno que la Universidad se enfoque también en eso y nosotros como ingenieros comerciales y los ingenieros industriales también le dan harto énfasis a esto y encuentro que esto es súper positivo, que este tipo de iniciativa de la universidad de potenciar a los alumnos en esta área". (E. 31, III). 


\section{Discusión y Conclusiones}

\section{Discusión}

La información analizada confirma el rol de las universidades en la formación de capital humano, conocimiento y capital emprendedor para dar soporte a la actividad emprendedora (Backs et al., 2019; Guerrero et al., 2016), así como en la formación de la actitud emprendedora del alumnado, y su potencial para constituirse en una plataforma de interacción entre el sector público y privado fomentando la actividad emprendedora y generando aprendizaje a partir de la implementación de nuevas rutinas y métodos (Guerrero y Urbano, 2012). Se ha observado que la implementación de este programa impulsa el desarrollo e implementación de un conjunto de nuevas prácticas y métodos psicopedagógicos, la formación de equipos docentes, el reclutamiento de estudiantes provenientes desde distintas facultades, el desarrollo de unidades de soporte y orientación en la universidad, y el compromiso de estudiantes, profesorado y autoridades en la implementación y éxito del proyecto. El programa fomenta la interacción de estudiantes, profesorado, empresas y sector público formando un ecosistema que facilita el aprendizaje. Esto permite afirmar que:

P1: La interacción entre el modelo de formación, el ecosistema de emprendimiento y estudiantes con distintos perfiles de ingreso dan forma a un ecosistema de aprendizaje.

La institución objeto de este estudio se encuentra entre las que asumen como objetivo desarrollar ecosistemas de emprendimiento como una de sus características distintivas (Guerrero et al., 2014). Coincidiendo con Backs et al. (2019), se observa la interacción de actores heterogéneos; dicha interacción permite el progreso más allá de la pura generación de proyectos de emprendimiento. En este caso el objetivo primordial era la formación de actitudes y competencias para innovar y emprender. La interacción de estudiantes con empresas y sector público no solamente potenció el desarrollo de sus proyectos de emprendimiento, sino también su motivación y actitud hacia aquellos. Esto confirma que la interacción de la institución educativa con su entorno social e industrial favorece la formación de ecosistemas de formación de talento, tal y como señalan recientemente Cao y Zhou (2018), y Hebles et al. (2019) que dichas interacciones son un soporte fundamental para formar la actitud emprendedora (Hebles et al., 2019). Esto permite afirmar que:

P2: El ecosistema de aprendizaje influye sobre la formación de la actitud emprendedora de los estudiantes.

Entre los hallazgos, destaca el papel determinante de un ecosistema de emprendimiento en la formación de la actitud emprendedora de las/os estudiantes, y la confirmación de que no se desarrolla de la misma manera con la mera implementación de un programa formativo. Es necesario reconocer la condición sistémica de los procesos formativos y tenerla en cuenta para el diseño del programa (Giovannella et al., 2015). El perfil de los estudiantes evoluciona con la implementación del programa y se confirma que este no afecta igual a cada estudiante; y que sus efectos son marginales y a veces negativos en estudiantes con altos niveles de exposición previa a la actividad emprendedora (Fayolle y Gailly, 2015). Esto explica por qué disminuyen las diferencias entre estudiantes más rezagados inicialmente y el resto, de manera que el discurso en términos de motivaciones, intereses, autoconfianza y orientación al logro se hace más homogéneo con el avance en el plan de estudios. La discusión anterior se resume en la siguiente proposición:

\section{P3: El desarrollo de la actitud emprendedora genera ajustes del perfil de los estudiantes.}

La literatura sobre formación emprendedora propone un portafolio de prácticas que pueden ser implementadas en este tipo de programas (Neck y Greene, 2011). Se ha confirmado la amplia heterogeneidad en el diseño de métodos y prácticas psicopedagógicas para la formación de 
actitudes emprendedoras (Kassean et al., 2015). Se observa que la integración de métodos enfocados al desarrollo de la creatividad, el fortalecimiento de habilidades como trabajar en equipo con métodos centrados en la planificación y desarrollo de proyectos genera resultados positivos (Chandler et al., 2011; García-Rodríguez et al., 2016). Este trabajo también confirma el importante papel que juegan los equipos de profesorado en el proceso (Raposo y do Paco, 2011), destacando la función orientadora de los mismos. Los ajustes del perfil de ingreso del alumnado demandan la flexibilidad de la institución educativa para adaptar e integrar las distintas opciones metodológicas disponibles. Dicha flexibilidad es un factor decisivo en la medida que alimenta el ecosistema de aprendizaje, mejora el ecosistema de emprendimiento y mantiene los niveles de motivación y compromiso de los estudiantes. Esto da soporte a las siguientes proposiciones:

P4: Los ajustes del perfil de los estudiantes influyen sobre la adaptación del modelo de enseñanza.

P5: Los ajustes del perfil de los estudiantes fortalece el ecosistema de emprendimiento.

La discusión y proposiciones desarrolladas en esta sección se resumen en la Figura 2.

Figura 2. Modelo de teorización

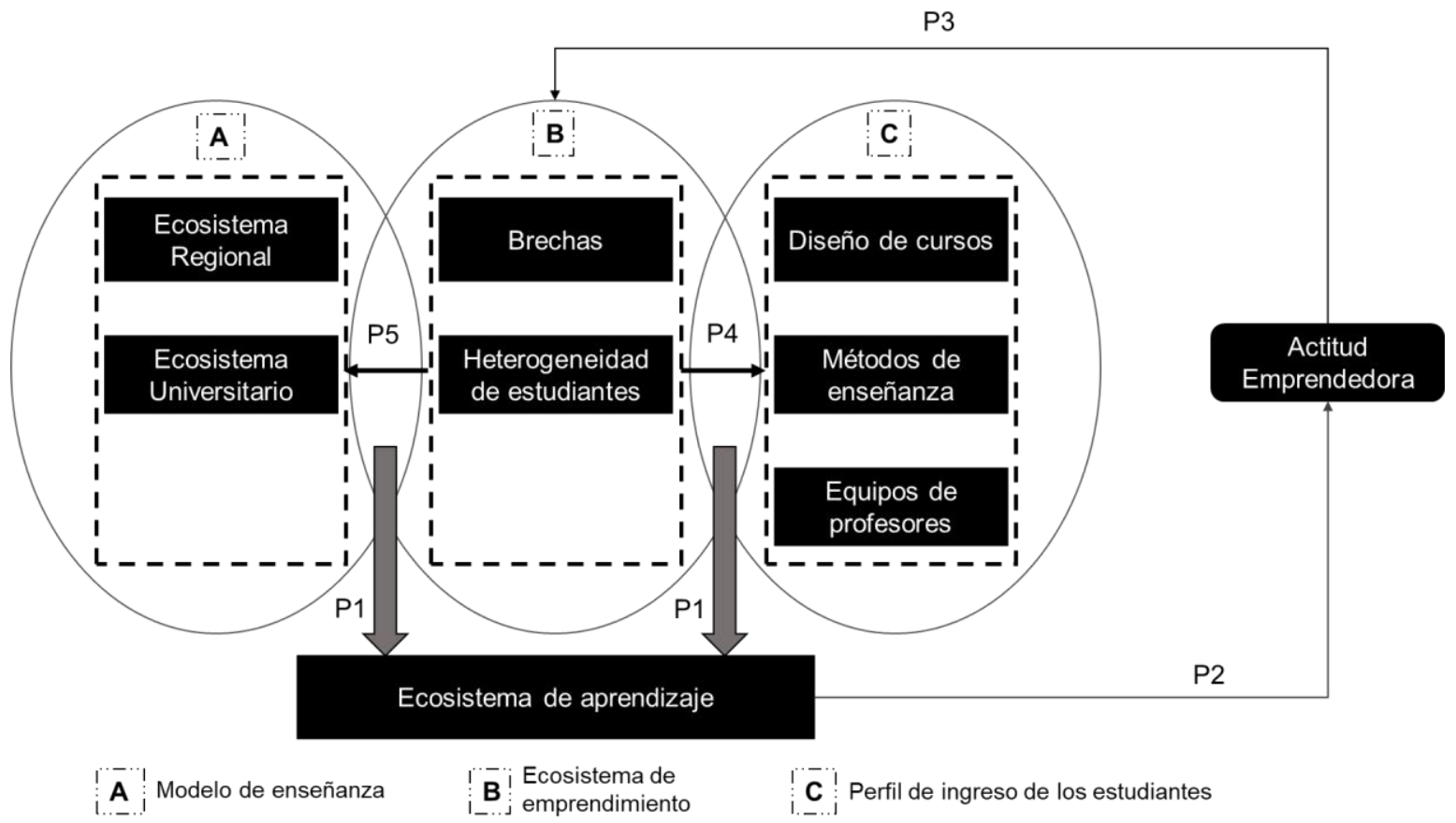

Fuente: Elaboración propia

Conclusiones, implicaciones y limitaciones

El objetivo de esta investigación ha sido determinar cómo influyen el ecosistema de emprendimiento, perfil de ingreso del alumnado y el diseño de un programa de enseñanza en la formación de la actitud emprendedora de estudiantes de pregrado. Basado en una investigación cualitativa de carácter exploratorio sobre el caso de la implementación de un programa de competencias en emprendimiento e innovación en una universidad, este trabajo propone que la interacción entre modelo de enseñanza, ecosistema de emprendimiento y estudiantes con 
distintos perfiles de ingreso dan forma a un ecosistema de aprendizaje. La dinámica de este ecosistema de aprendizaje sería el principal determinante para el desarrollo de la actitud emprendedora de las y los estudiantes que participan en el programa. Estos hallazgos coinciden con investigaciones previas que destacan la importancia de ecosistemas que conecten a las universidades con el sector público y privado para fomentar la innovación y el emprendimiento (Guerrero et al., 2014; Guerrero et al., 2016). También confirman la importancia de desarrollar vínculos sólidos en la triple hélice universidad-empresa-instituciones públicas para favorecer la formación de ecosistemas generadores de talento (Cao y Zhou, 2018).

Este trabajo destaca la visión sistémica del proceso formativo de la actitud y competencia para emprender. Se asume la importancia de los aspectos curriculares y metodológicos, y de considerar la heterogeneidad en las competencias de entrada y en las motivaciones de los estudiantes (Lans et al., 2013; Nabi et al., 2017; Suárez-Ortega et al., 2020) en una orientación personalizada e integral. Se destaca la relevancia del efecto interacción entre estos factores y el ecosistema de emprendimiento. Se propone que el proceso formativo es continuo y que se retroalimenta de los resultados en la formación de la actitud emprendedora de las/os estudiantes, producto de la implementación del programa en etapas tempranas. Por eso se sugiere que la flexibilidad de la institución educativa es fundamental para el fortalecimiento del ecosistema de aprendizaje y el desarrollo de la actitud de emprender del alumnado. Esto significa que la universidad debe ser capaz de ajustar las metodologías y técnicas pedagógicas, así como los equipos docentes y los mecanismos de vinculación con el ecosistema de emprendimiento regional.

Específicamente en lo que se refiere al modelo de aprendizaje, se observa consenso entre los actores implicados en valorar la variedad de métodos y técnicas de enseñanza utilizadas. El alumnado valora particularmente la posibilidad de trabajar en el terreno y en contacto con la comunidad, clientes y usuarios de servicios, resolviendo problemas conjuntamente. Directivos y profesorado destacan la coherencia de la propuesta metodológica y del proyecto de intervención. Valoran positivamente que el programa se inicie con cursos enfocados principalmente a desarrollar carencias actitudinales, así como la implementación de actividades concretas y prácticas en el contexto empresarial y social donde se ubica la universidad. Se observa que esto permite que las y los estudiantes hagan una transición adecuada desde despertar el interés por el emprendimiento, hasta desarrollar la actitud y generar un comportamiento emprendedor diseñando e implementando proyectos en la comunidad. Así se integran métodos orientados a la experimentación con otros métodos académicamente más tradicionales (Chandler et al., 2011; Kassean et al., 2015; Neck y Greene, 2011).

Se observó que el perfil de ingreso era heterogéneo y existían carencias y limitaciones que había que superar. Una parte del alumnado provenía de ambientes vulnerables, y presentaba problemas de personalidad y autoestima. Se diferenciaban en carreras de procedencia, intereses, capital cultural, habilidades interpersonales, liderazgo y capacidad de trabajar en equipo. Coincidiendo con Keinanen y Kairisto-Mertanen (2019) se observó que la gran importancia que el profesorado otorgó a conocer dichas limitaciones y la heterogeneidad de los grupos es muy relevante para plantear el proceso formativo de forma efectiva. Confirmando lo señalado por Lans et al. (2013), las diferencias culturales y de disciplinas de procedencia, por una parte, fueron obstaculizadores del proceso formativo, pero, por otra, también generaron un efecto positivo enriqueciendo el aprendizaje, principalmente los procesos de generación y desarrollo de ideas.

Finalmente se observa que la implementación del proyecto impulsa la formación de un conjunto de vínculos dentro de la universidad y con el entorno público y privado que dan forma a un ecosistema de emprendimiento. La interacción entre estos actores mejora la capacidad del alumnado para identificar oportunidades de emprendimiento, así como para buscar fuentes de apoyo para llevar a cabo sus propuestas. Coincidiendo con Alda-Varas et al. (2012), esto confirmaría la importancia que tiene desarrollar habilidades de exploración del entorno para el éxito del proceso emprendedor. Asimismo, refuerza la idea sobre el rol central que pueden jugar las universidades en la formación de ecosistemas de emprendimiento (Backs et al., 2019; 
Guerrero et al., 2016). Por último, se entrega soporte adicional a la idea de que la interacción universidad-empresa-instituciones públicas no solamente beneficia la actividad emprendedora, sino que también es clave para el fortalecimiento de los procesos formativos en emprendimiento (Cao y Zhou, 2018; Hebles et al., 2019).

En definitiva, este trabajo contribuye a la orientación para el desarrollo profesional aportando conocimiento sobre formación emprendedora (Bae et al., 2014; Fayolle, 2013; Morris et al., 2013), sobre la formación de la actitud y la competencia de emprender (Fayolle y Gailly, 2015; Harris y Gibson, 2008), y a la comprensión del papel que pueden jugar las universidades tanto en procesos formativos en emprendimiento como en la formación y fortalecimiento de ecosistemas de emprendimiento (Link y Sarala, 2019; Pedroza-Zapata y Silva-Flores, 2020; Stam, 2015).

Finalmente, es necesario reconocer las limitaciones de esta investigación. El diseño utilizado y el carácter cualitativo y exploratorio de este trabajo limita la generalización de los resultados. Otra limitación son sesgos propios en la interpretación y análisis de los datos por parte de los investigadores también derivados del tipo de estudio. Se ha buscado controlar dichas limitaciones con la aplicación rigurosa de los métodos descritos. Las conclusiones obtenidas para el caso estudiado suponen una primera aportación que se completará con sucesivos estudios de caso en profundidad. Estudios cualitativos adicionales contribuirán aumentando la muestra, así como también validando la herramienta y método utilizado. Las proposiciones podrían ser testeadas con otros diseños y, eventualmente, con análisis de fiabilidad y validez estadística que complete la información obtenida en la aplicación del software de análisis de datos utilizado (Llanos-Contreras et al., 2019). Particularmente útil en este caso, estudios basados en encuestas.

\section{Referencias bibliográficas}

Ajzen, I. y Fishbein, M. (2000). Attitudes and the attitude-behavior relation: Reasoned and automatic processes. European Review of Social Psychology, 11(1), 1-33. https://doi.org/10.1080/14792779943000116

Alda-Varas, R., Villardón-Gallego, L. y Elexpuru-Albizuri, I. (2012). Propuesta y validación de un perfil de competencias de la persona emprendedora. Implicaciones para la formación. Electronic Journal of Research in Educational Psychology, 10(3), 1057-1080. http://dx.doi.org/10.25115/ejrep.v10i28.1549

Alda, R. F. (2010). Perfil emprendedor de los estudiantes de la Facultad de Economía y Administración, de la Universidad Católica del Norte, Antofagasta, Chile. [Tesis Doctoral, Universidad de Deusto]. https://dkh.deusto.es/comunidad/thesis/recurso/perfilemprendedor-de-los-estudiantes-de-la/e7c46839-1a94-423c-9e05-38a9d997fc1e

Alonso-Dos-Santos, M. y Llanos-Contreras, O. (2018). Family business performance in a postdisaster scenario: The influence of socioemotional wealth importance and entrepreneurial orientation. Journal of Business Research, 101, 492-498. https://doi.org/doi:10.1016/j.jbusres.2018.12.057

Azqueta, A. y Naval, C. (2019). Educación para el emprendimiento - Entrepreneurship education: Una propuesta para el desarrollo humano. Revista Española de Pedagogía, 77(274), 517534. https://doi.org/10.22550/REP77-3-2019-03 
Backs, S., Gunther, M. y Stummer, C. (2019). Stimulating academic patenting in a university ecosystem: An agent-based simulation approach. Journal of Technology Transfer, 44(2), 434-461. https://doi.org/10.1007/s10961-018-9697-x

Bae, T. J., Qian, S., Miao, C. y Fiet, J. O. (2014). The Relationship between Entrepreneurship Education and Entrepreneurial Intentions: A Meta-Analytic Review. Entrepreneurship Theory and Practice, 38(2), 217-254. https://doi.org/10.1111/etap.12095

Báez, J. y De Tudela, P. (2007). Investigación cualitativa: Esic Editorial.

Cao, Z. P. y Zhou, M. (2018). Research on the Innovation and Entrepreneurship Education Mode in Colleges and Universities Based on Entrepreneurial Ecosystem Theory. Educational Sciences-Theory \& Practice, 18(5), 1612-1619. https://doi.org/10.12738/estp.2018.5.060

Chandler, G. N., DeTienne, D. R., McKelvie, A. y Mumford, T. V. (2011). Causation and effectuation processes: A validation study. Journal of Business Venturing, 26(3), 375-390. https://doi.org/10.1016/j.jbusvent.2009.10.006

De Massis, A. y Kotlar, J. (2014). The case study method in family business research: Guidelines for qualitative scholarship. Journal of Family Business Strategy, 5(1), 15-29. https://doi.org/10.1016/i.jfbs.2014.01.007

Díaz-García, C., Sáez-Martínez, F. y Jiménez-Moreno, J. (2015). Evaluación del impacto del programa educativo "Emprendedores" en la intención emprendedora de los participantes. RUSC. Universities and Knowledge Society Journal, 12(3), 17-31. https://doi.org/ $\underline{10.7238 / \text { rusc.v12i3.2146 }}$

Fayolle, A. (2013). Personal views on the future of entrepreneurship education. Entrepreneurship \& Regional Development, 25(7-8), 692-701. https://doi.org/10.1080/08985626.2013.821318

Fayolle, A. y Gailly, B. (2015). The impact of entrepreneurship education on entrepreneurial attitudes and intention: Hysteresis and persistence. Journal of Small Business Management, 53(1), 75-93. https://doi.org/10.1111/jsbm.12065

García-Rodríguez, F. J., Ruiz-Rosa, C.-I., Gil-Soto, E. y Gutiérrez-Taño, D. (2016). Promoting entrepreneurship education among university students: Design and evaluation of an intervention programme / Fomento de la educación emprendedora entre el alumnado universitario: Diseño y evaluación de un programa de intervención. Cultura y Educación, 28(3), 565-600. https://doi.org/10.1080/11356405.2016.1196897

Giovannella, C., Andone, D., Dascalu, M., Popescu, E., Rehm, M. y Roccasalva, G. (2015). Smartness of learning ecosystems and its bottom-up emergence in six European campuses. Interaction Design and Architectures, 27(5), 79-92.

Guerrero, M. y Urbano, D. (2012). The development of an entrepreneurial university. Journal of Technology Transfer, 37(1), 43-74. https://doi.org/10.1007/s10961-010-9171-x

Guerrero, M., Urbano, D., Cunningham, J. y Organ, D. (2014). Entrepreneurial universities in two European regions: A case study comparison. Journal of Technology Transfer, 39(3), 415434. https://doi.org/10.1007/s10961-012-9287-2

Guerrero, M., Urbano, D., Fayolle, A., Klofsten, M. y Mian, S. (2016). Entrepreneurial universities: Emerging models in the new social and economic landscape. Small Business Economics, 47(3), 551-563. https://doi.org/10.1007/s11187-016-9755-4

Hägg, G. y Kurczewska, A. (2020). Guiding the student entrepreneur - Considering the emergent adult within the pedagogy-andragogy continuum in entrepreneurship education. Education \& Training (London), 62(7/8), 759-777. 
Harris, M. L. y Gibson, S. G. (2008). Examining the entrepreneurial attitudes of US business students. Education $+\quad$ Training, 50(7), https://doi.org/10.1108/00400910810909036

Hebles, M., Llanos-Contreras, O. y Yániz-Álvarez-de-Eulate, C. (2019). Perceived evolution of the entrepeneurial competence based on the implementation of a training program in entrepreneurship and innovation. Revista Española de Orientacion y Psicopedagogia, 30(1), 9-26. https://doi.org/10.5944/reop.vol.30.num.1.2019.25191

Holmgren, C., From, J., Olofsson, A., Karlsson, H., Snyder, K. y Sundtröm, U. (2004). Entrepreneurship education: Salvation or damnation? Journal of Entrepreneurship Education, 8, 7-19.

Hunter, J. D. y Cooksey, R. W. (2004). The decision to outsource: a case study of the complex interplay between strategic wisdom and behavioural reality. Journal of the Australian and New Zealand Academy of Management, 10(2), 26-40.

Huq, A. y Gilbert, D. (2017). All the world's a stage: Transforming entrepreneurship education through design thinking. Education + Training, 59(2), 155-170. https://doi.org/10.1108/ET$\underline{12-2015-0111}$

Kassean, H., Vanevenhoven, J., Liguori, E. y Winkel, D. E. (2015). Entrepreneurship education: A need for reflection, real-world experience, and action. International Journal of Entrepreneurial Behavior \& Research, 21(5), 690-708. https://doi.org/10.1108/ijebr-07$\underline{2014-0123}$

Keinanen, M. M. y Kairisto-Mertanen, L. (2019). Researching learning environments and students' innovation competences. Education and Training, 61(1), 17-30. https://doi.org/10.1108/et03-2018-0064

Krauss, C. (2011). Actitudes emprendedoras de los estudiantes universitarios: El caso de la Universidad Católica del Uruguay. Dimensión empresarial, 9(1), 28-40.

Kuratko, D. F. (2005). The emergence of entrepreneurship education: Development, trends, and challenges. Entrepreneurship Theory and Practice, 29(5), 577-598. https://doi.org/10.1111/i.1540-6520.2005.00099.x

Lans, T., Oganisjana, K., Taks, M. y Popov, V. (2013). Learning for entrepreneurship in heterogeneous groups: Experiences from an international, interdisciplinary higher education student programme. Trames-Journal of the Humanities and Social Sciences, 17(4), 383-399. https://doi.org/10.3176/tr.2013.4.05

Link, A. N. y Sarala, R. M. (2019). Advancing conceptualisation of university entrepreneurial ecosystems: The role of knowledge-intensive entrepreneurial firms. International Small Business Journal: Researching Entrepreneurship, 37(3), 289-310. https://doi.org/10.1177/0266242618821720

Llanos-Contreras, O., Jabri, M. y Sharma, P. (2019). Temporality and the role of shocks in explaining changes in socioemotional wealth and entrepreneurial orientation of small and medium family enterprises. International Entrepreneurship and Management Journal, 5(4), 1269-1289. https://doi.org/10.1007/s11365-019-00595-4

Llanos-Contreras, O. A. y Jabri, M. (2019). Exploring family business decline with socioemotional wealth perspective. Academia-Revista Latinoamericana de Administración, 32(1), 63-78. https://doi.org/10.1108/arla-02-2018-0042 
Morris, M. H., Webb, J. W., Fu, J. y Singhal, S. (2013). A competency-based perspective on entrepreneurship education: Conceptual and empirical insights. Journal of Small Business Management, 51(3), 352-369. https://doi.org/10.1111/jsbm.12023

Nabi, G., Liñán, F., Fayolle, A., Krueger, N. y Walmsley, A. (2017). The impact of entrepreneurship education in higher education: A systematic review and research agenda. Academy of

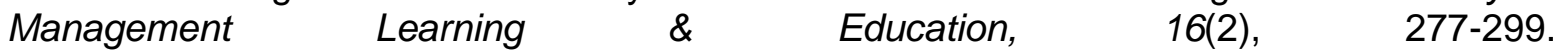
https://doi.org/10.5465/amle.2015.0026

Neck, H. M. y Greene, P. G. (2011). Entrepreneurship education: Known worlds and new frontiers. Journal of Small Business Management, 49(1), 55-70. https://doi.org/10.1111/j.1540$\underline{627 X .2010 .00314 . x}$

Palomares-Montero, D., Chisvert-Tarazona, M. J. y Suárez-Ortega, M. (2019). Formación y orientación para el emprendimiento. Lo que dicen la bibliometría y los emprendedores noveles. Revista Española de Orientación y Psicopedagogía, 30(1), 131-149. https://doi.org/10.5944/reop.vol.30.num.1.2019.25198

Pedroza-Zapata, A. R. y Silva-Flores, M. L. (2020). Ecosistema Universitario de Ciencia, Tecnología, Innovación y Emprendimiento. Magis, Revista Internacional de Investigación en Educación, 12(25), 93-110. https://doi.org/10.11144/Javeriana.m12-25.euct

Popov, V., Brinkman, D., Biemans, H. J. A., Mulder, M., Kuznetsov, A. y Noroozi, O. (2012). Multicultural student group work in higher education: An explorative case study on challenges as perceived by students. International Journal of Intercultural Relations, 36(2), 302-317. https://doi.org/10.1016/j.ijintrel.2011.09.004

Radharamanan, R. y Juang, J. N. (2012). Innovation and entrepreneurship in engineering education at MUSE. Journal of the Chinese Institute of Engineers, 35(1), 25-36. https://doi.org/10.1080/02533839.2012.624797

Raposo, M. y do Paco, A. (2011). Entrepreneurship education: Relationship between education and entrepreneurial activity. Psicothema, 23(3), 453-457.

Raposo, M., do Paço, A. y Ferreira, J. (2008). Entrepreneur's profile: A taxonomy of attributes and motivations of university students. Journal of Small Business and Enterprise Development, 15(2), 405-418. https://doi.org/10.1108/14626000810871763

Sánchez-García, J. C. y Hernández-Sánchez, B. (2016). Influencia del Programa Emprendedor Universitario (PREU) para la mejora de la actitud emprendedora. Pampa: Revista $\begin{array}{llll}\text { Interuniversitaria de } \quad \text { Estudios } & \text { Territoriales, }\end{array}$ https://doi.org/10.1108/00400911011017708

Sánchez, J. C. (2011). University training for entrepreneurial competencies: Its impact on intention of venture creation. International Entrepreneurship and Management Journal, 7(2), 239254. https://doi.org/10.1007/s11365-010-0156-x

Schmidt, J. J. y Molkentin, K. F. (2015). Building and maintaining a regional inter-university ecosystem for entrepreneurship: Entrepreneurship education consortium. Journal of Entrepreneurship Education, 18(1), 157-168.

Starman, A. B. (2013). The case study as a type of qualitative research. Journal of Contemporary Educational Studies/Sodobna Pedagogika, 64(1), 29-35.

Suárez Ortega, M., Sánchez-García, M. F. y Soto-González, M. D. (2020). Desarrollo de la carrera emprendedora: Identificación de perfiles, competencias y necesidades. Revista Complutense de Educación 31(2) (2020): 173-84. https://doi.org/10.5209/rced.62001 
Stam, E. (2015). Entrepreneurial ecosystems and regional policy: A sympathetic critique. European Planning Studies, 23(9), 1759-1769. https://doi.org/10.1080/09654313.2015.1061484

Thomas, D. R. (2006). A General Inductive Approach for Analyzing Qualitative Evaluation Data. American Journal of Evaluation, 27(2), 237-246. https://doi.org/10.1177/1098214005283748

Vanevenhoven, J. (2013). Advances and challenges in entrepreneurship education. Journal of Small Business Management, 51(3), 466-470. https://doi.org/10.1111/isbm.12043

Woodside, A. G. (2010). Case study research: Theory, methods, and practice. Emerald Group Publishing.

Yin, R. K. (2014). Case study research: design and methods (5th ed.). SAGE.

Fecha de entrada: 06 julio 2020 Fecha de revisión: 18 diciembre 2020 Fecha de aceptación: 23 diciembre 2020 